\title{
The Antimalaria Agent Artemisinin Exerts Antiangiogenic Effects in Mouse Embryonic Stem Cell-Derived Embryoid Bodies
}

\author{
Maria Wartenberg, Sandra Wolf, Paula Budde, Frank Grünheck, Helmut Acker, \\ Jürgen Hescheler, Gerda Wartenberg, and Heinrich Sauer
}

\author{
Department of Neurophysiology and Center for Molecular Medicine (MW, SW, PB, FG, JH, GW, HS), University of \\ Cologne, Cologne, and Max-Planck-Institute for Molecular Physiology (HA), Dortmund, Germany
}

\begin{abstract}
SUMMARY: Artemisinin is widely used as an agent to treat malaria; the possible antiangiogenic effects of this compound are unknown. In the present study, the antiangiogenic effects of artemisinin were investigated in mouse embryonic stem cell-derived embryoid bodies, which are a model system for early postimplantation embryos and which efficiently differentiate capillaries. Artemisinin dose dependently inhibited angiogenesis in embryoid bodies and raised the level of intracellular reactive oxygen species. Furthermore impaired organization of the extracellular matrix component laminin and altered expression patterns of matrix metalloproteinases 1, 2, and 9 were observed during the time course of embryoid body differentiation. Consequently accelerated penetration kinetics of the fluorescent anthracycline doxorubicin occurred within the tissue, indicating increased tissue permeability. Artemisinin down-regulated hypoxia-inducible factor- $1 \alpha$ and vascular endothelial growth factor (VEGF) expression, which control endothelial cell growth. The antiangiogenic effects and the inhibition of hypoxia-inducible factor- $1 \alpha$ and VEGF were reversed upon cotreatment with the free radical scavengers mannitol and vitamin E, indicating that artemisinin may act via reactive oxygen species generation. Furthermore, capillary formation was restored upon coadministration of exogenous VEGF. The data of the present study suggest that the antiangiogenic activity of artemisinin and the increase in tissue permeability for cytostatics may be exploited for anticancer treatment. (Lab Invest 2003, 83:1647-1655).
\end{abstract}

$T$ he sesquiterpene artemisinin is a drug isolated from decoctions of leaves of Artemisia annua and has been used in traditional Chinese medicine for the treatment of fevers for more than 2000 years (Meshnick et al, 1996). During recent years this compound and several derivatives have emerged as the most potent antimalaria agents, being especially active against chloroquine-resistant malaria strains (Haynes, 2001). Furthermore, artemisinin and its derivatives have been demonstrated to exert antitumor activity in several in vitro studies, suggesting that in the future this compound may be used in anticancer strategies (Efferth et al, 2001; Posner et al, 1999). The mode of action of artemisinin on plasmodium falciparum has been associated with the generation of oxidative stress by this compound, because the intraerythrocytic activation of the drug peroxide bond by iron(II)-heme produced during hemoglobin degradation should generate reactive oxygen species (ROS). However, it has been recently speculated that parasite

\section{DOI: 10.1097/01.LAB.0000098424.38003.FF}

Received September 3, 2003.

This work was supported by the Competence Network Stem Cell Research, Northrhine-Westfalia, and the Cologne Fortune Programme, University of Cologne.

Address reprint requests to: Dr. H. Sauer, Department of Neurophysiology, University of Cologne, Robert-Koch-Str. 39, D-50931 Cologne, Germany. E-mail: heinrich.sauer@uni-koeln.de death in the presence of artemisinin is presumably not a result of nonspecific or random cell damage caused by freely diffusing ROS but might involve specific radicals and targets that have yet to be identified at the molecular level (Meshnick, 1994; Robert et al, 2002).

The possible antiangiogenic potential of artemisinin has not been investigated. Inhibition of tumor-induced angiogenesis has proven one of the most promising anticancer strategies, requiring the need for the development of efficient novel antiangiogenic agents (Molema, 2002). However, inhibition of blood vessel growth may also cause teratogenic effects in growing embryos, excluding the usage of antiangiogenic agents in pregnant women. In this respect derivatives of artemisinin, ie, dihydroartemisinin and artesunate, have been shown to exert contragestational effect. In mice and in rabbits, these agents caused embryo absorption, whereas in hamsters and guinea pigs they induced abortion (Xu and Zhang, 1996).

In the present study, we used embryoid bodies derived from mouse embryonic stem (ES) cells to investigate the possible antiangiogenic effects of artemisinin. Embryoid bodies develop a capillary network (Vittet et al, 1996; Wartenberg et al, 1998) and have been previously shown to be suitable for the assessment of the activity of antiangiogenic (Sauer et al, 2000; Wartenberg et al, 1998) as well as embryotoxic compounds (Seiler et al, 2002). We demon- 
strated that artemisinin significantly inhibited vasculogenesis/angiogenesis in ES cell-derived embryoid bodies by a mechanism that involves the generation of free oxygen radicals.

\section{Results}

\section{Antiangiogenic Effects of Artemisinin}

To investigate the possible antiangiogenic effects of artemisinin, embryoid bodies were treated from Day 3 to Day 8 with increasing concentrations of artemisinin ranging from 0.5 to $50 \mu \mathrm{m}$. Cell areas positive for the endothelial cell-specific marker platelet endothelial cell adhesion molecule-1 (PECAM-1; CD31) were assessed (Fig. 1). In parallel the growth of embryoid bodies and differentiation of cardiac cell areas was assessed in the presence of either 10 or $50 \mu \mathrm{M}$ artemisinin (Fig. 2, A to C). In a further set of experiments, the effects of coadministration of the nonspecific free radical scavenger vitamin $\mathrm{E}(50 \mu \mathrm{M})$ as well as the hydroxyl radical-specific scavenger mannitol (10 $\mathrm{mm}$ ) on angiogenesis were investigated (Fig. 3). As shown in Figure 1, artemisinin dose dependently inhibited angiogenesis in embryoid bodies $(n=3)$. Determination of PECAM-1-positive cell areas in optical tissue sections of $100 \mu \mathrm{M}$ thickness revealed a significant antiangiogenic effect of artemisinin, which became apparent at $0.5 \mu \mathrm{M}$ and was maximum at concentrations of 10 and $50 \mu \mathrm{M}$. Artemisinin at concentrations of 10 and $50 \mu \mathrm{M}$ slowed the growth of embryoid bodies (Fig. 2A) and decreased the expression of the proliferation-associated antigen Ki-67 (Fig. 2B) $(n=3)$, although no cytotoxic effect, as evaluated by the lethal cell dye Sytox-Green, was observed (data not shown). No impairment of differentiation of cardiac cell areas was observed $(n=3)$, indicating that artemisinin did not generally inhibit differentiation of the mesodermal cell lineage (Fig. 2C). Interestingly, significant difference between the growth kinetics of the untreated control and the samples treated with arte-

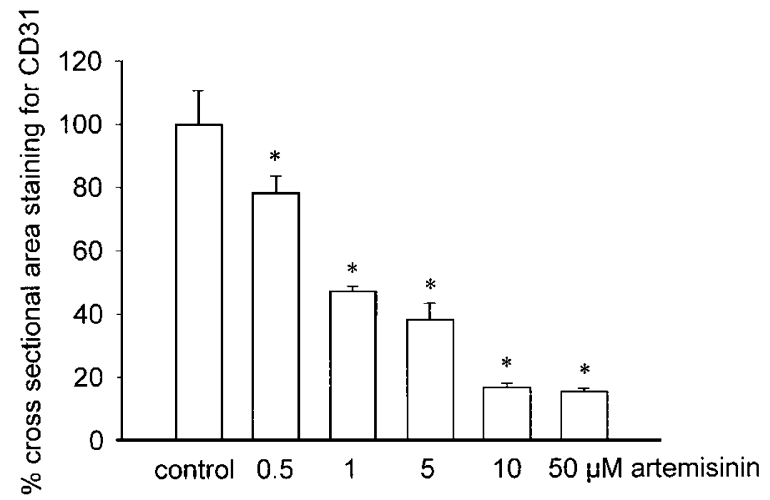

Figure 1.

Dose-response curve of the antiangiogenic effect of artemisinin. Embryoid bodies were treated from Day 3 through Day 8 with artemisinin in concentrations of $0.5,1,5,10$, and $50 \mu \mathrm{M}$ artemisinin. Cell areas positive for platelet endothelial cell adhesion molecule-1 (PECAM-1; CD31) were evaluated in optical sections of $100-\mu \mathrm{m}$ thickness by confocal laser scanning microscopy. Note that artemisinin exerted a dose-dependent antiangiogenic effect.
A

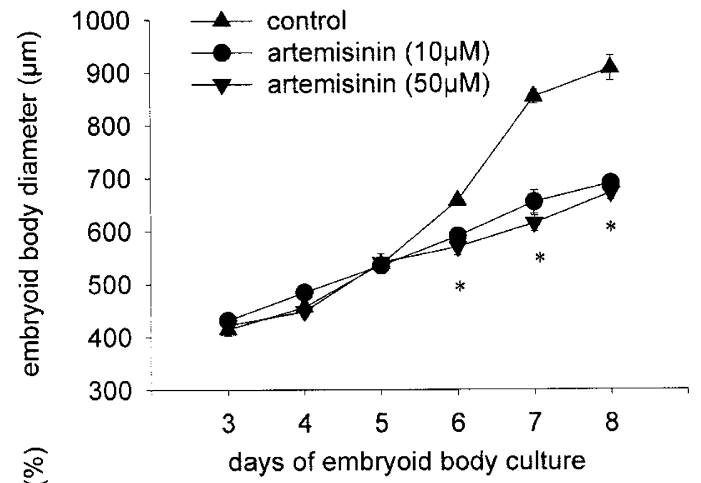

B

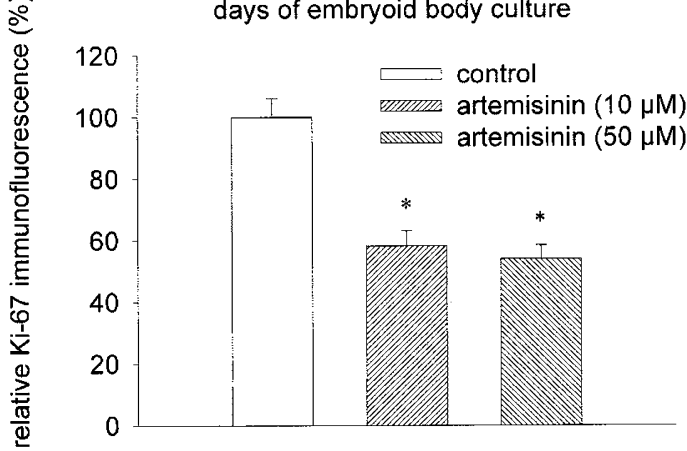

$\mathrm{C}$

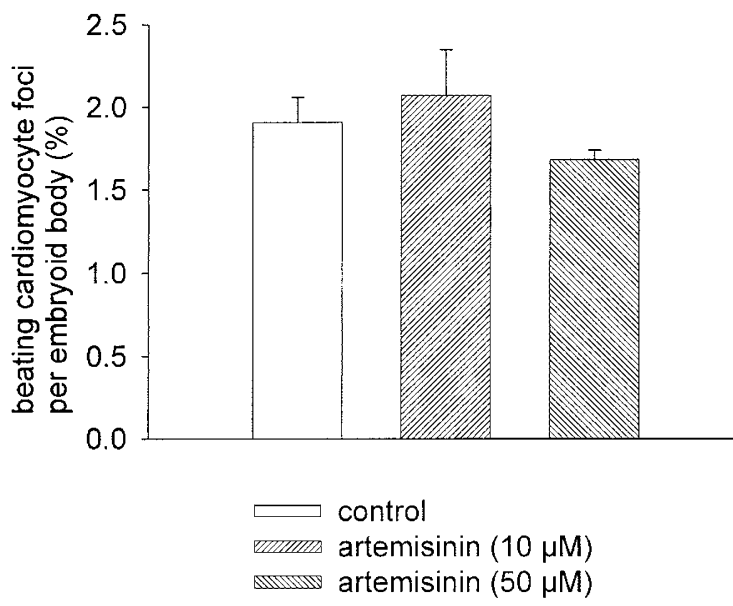

Figure 2.

Effects of artemisinin on the growth of embryoid bodies (A), the expression of the proliferation marker Ki-67 (B), and the differentiation of cardiac cells (C). Embryoid bodies were treated from Day 3 through Day 8 with either 10 or 50 $\mu \mathrm{M}$ artemisinin, and diameters were determined every 24 hours by visual inspection (A). Ki-67 immunostaining was performed on Day 8 of cell culture, ie, after a 5-day treatment with artemisinin (B). After plating to Petriperm Petri dishes on Day 6 of cell culture, embryoid bodies started spontaneous contractions within 1 to 2 days. On Day 9 of cell culture, $100 \%$ of embryoid bodies in the control and in the artemisinin-treated samples exerted spontaneous contractions arising from at least one focus of cardiomyocytes, which were analyzed by visual inspection of cell contraction. Note that artemisinin treatment did not significantly change the number of beating cardiomyocyte foci per embryoid body (C).

misinin was not observed before Day 6, which is the time when vasculogenesis/angiogenesis in embryoid bodies occurs (Wartenberg et al, 1998). The antiangiogenic effects of 10 and $50 \mu \mathrm{M}$ artemisinin were nearly completely reversed upon coadministration of the nonspecific free radical scavenger vitamin $\mathrm{E}(50 \mu \mathrm{M})$ 

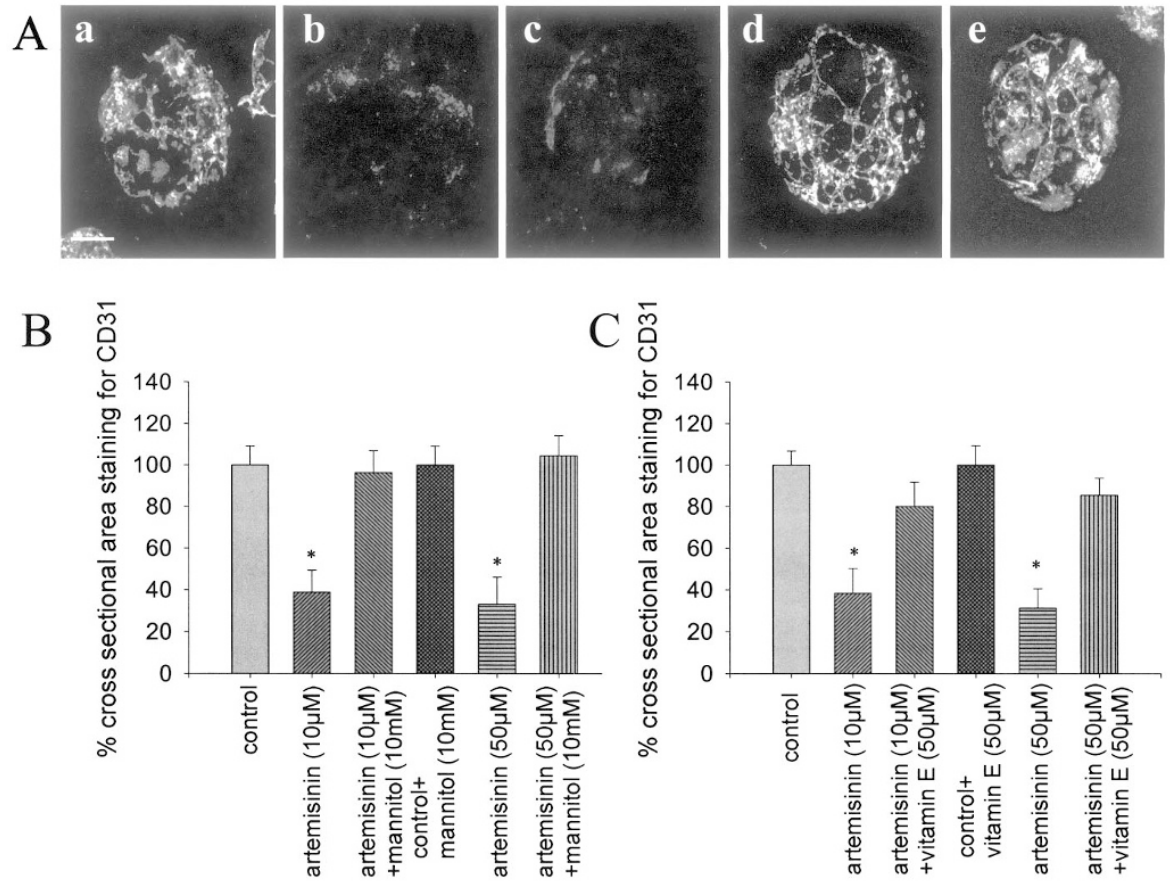

Figure 3.

Antiangiogenic effects of artemisinin and reversal by the free radical scavengers mannitol (10 mm) and vitamin $\mathrm{E}(50 \mu \mathrm{M})$. Embryoid bodies remained untreated (control) or were treated from Day 3 through Day 8 with either 10 or $50 \mu \mathrm{m}$ artemisinin in the absence or presence of either mannitol or vitamin E. A, Representative embryoid bodies stained with an antibody directed against the endothelial cell marker PECAM-1 (CD31) - a, untreated control; b, embryoid body treated with 10 $\mu \mathrm{M}$ artemisinin; c, embryoid body treated with $50 \mu \mathrm{M}$ artemisinin; d, embryoid body treated with $50 \mu \mathrm{m}$ artemisinin in the presence of 10 mM mannitol; e, embryoid body treated with $50 \mu \mathrm{m}$ artemisinin in the presence of vitamin $\mathrm{E}$. The bar represents $100 \mu \mathrm{M}$. B and C, quantitative evaluation of the cross-sectional area staining positive for CD31 in three independent experiments under experimental conditions as indicated. In each experiment at least 20 embryoid bodies were analyzed. ${ }^{*} p$ $<0.05$, significantly different from the untreated control.

and the hydroxyl radical scavenger mannitol (10 mm) (n = 3) (Fig. 3).

\section{Down-Regulation of Hypoxia-Inducible Factor-1a

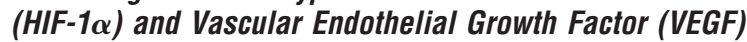 upon Treatment of Embryoid Bodies with Artemisinin}

Vascular growth is dependent on the presence of VEGF, which activates endothelial cell proliferation by binding to either VEGF receptor 1 (flt-1) or VEGF receptor 2 (flk-1/KDR). VEGF expression is under the control of the transcription factor HIF-1, which is activated under hypoxic conditions and degraded under normoxia (Mazure et al, 2003). To investigate the effects of artemisinin on HIF- $1 \alpha$ (Fig. 4, A and B) and VEGF (Fig. 5, A and B) expression, embryoid bodies remained either untreated or were treated with either 10 or $50 \mu \mathrm{m}$ artemisinin. In parallel experiments embryoid bodies were treated either with the free radical scavengers vitamin $\mathrm{E}(50 \mu \mathrm{M})$ or mannitol (10 $\mathrm{mm}$ ) alone or in combination with artemisinin. Upon treatment with either 10 or $50 \mu \mathrm{M}$ artemisinin, HIF-1 $\alpha$ was down-regulated to $64 \pm 2 \%$ and $63 \pm 3 \%$, respectively (untreated control set to $100 \%)(n=3)$. VEGF expression was significantly down-regulated to $40 \pm 6 \%$ and $42 \pm 3 \%$ upon treatment with 10 and 50 $\mu \mathrm{M}$ artemisinin, respectively $(n=3)$. Down-regulation of HIF- $1 \alpha$ and VEGF was significantly reduced in the presence of either vitamin $\mathrm{E}$ or mannitol, suggesting that the antiangiogenic effects of artemisinin are a result of redox-mediated reduction of VEGF expression.

To validate the assumption that the antiangiogenic effects of artemisinin were owing to down-regulation of VEGF, additional experiments were performed in which angiogenesis was stimulated with $50 \mathrm{ng} / \mathrm{ml}$ VEGF in either the absence or presence of 10 and 50 $\mu \mathrm{M}$ artemisinin, respectively (Fig. 6). We observed that VEGF alone stimulated angiogenesis in embryoid bodies, which resulted in significantly increased cell areas staining positive for CD31 $(n=3)$. Furthermore the antiangiogenic effect of artemisinin was significantly reversed in the presence of VEGF, which demonstrates that the compound acts via down-regulation of $\operatorname{VEGF}(n=3)$.

\section{Generation of ROS in Embryoid Bodies upon Treatment with Artemisinin}

The possible mode of action of artemisinin on the plasmodium parasite has been attributed to the generation of active peroxides that kill intraerythrocytic Plasmodium by interacting with the heme discarded by proteolysis of ingested hemoglobin (Jefford, 2001). Because our experiments demonstrated that the antiangiogenic effects of artemisinin were reversed upon coadministration of free radical scavengers, we investigated the generation of ROS by this compound using the redox-sensitive dye 2'7'-dichlorodihydrofluorescein diacetate $\left(\mathrm{H}_{2} \mathrm{DCF}-\mathrm{DA}\right)$, which is converted to a fluores- 

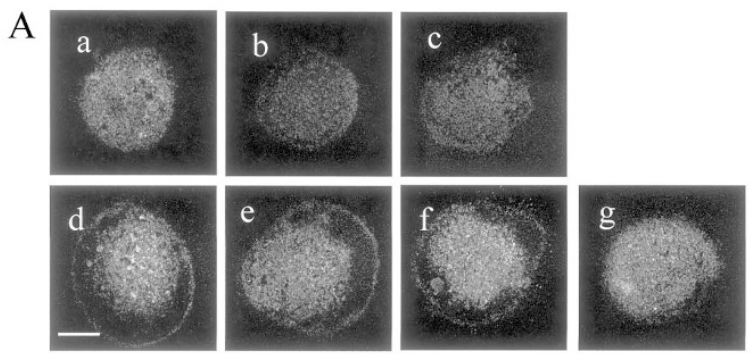

$\mathrm{B}$

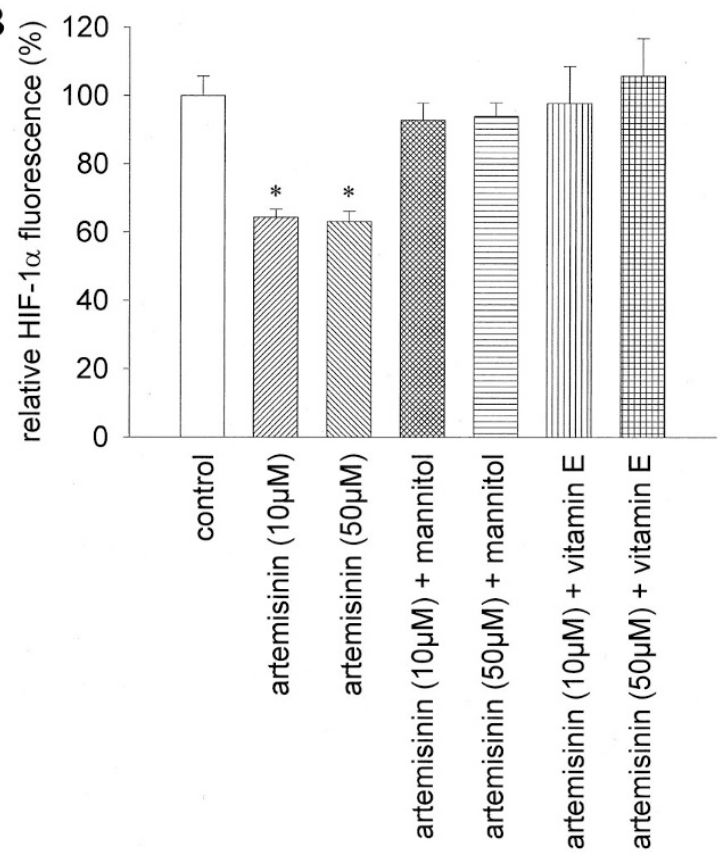

Figure 4.

Down-regulation of hypoxia-inducible factor- $1 \alpha$ (HIF-1 $\alpha$ ) expression upon treatment with artemisinin and effects of free radical scavengers. A, Representative embryoid bodies that remained untreated (control) (a) or were treated with $10 \mu \mathrm{m}$ artemisinin (b), $50 \mu \mathrm{m}$ artemisinin (c), $10 \mathrm{~mm}$ mannitol alone (d), $50 \mu \mathrm{m}$ vitamin $\mathrm{E}$ alone (e), $50 \mu \mathrm{m}$ artemisinin in the presence of 10 mM mannitol (f), or $50 \mu \mathrm{M}$ artemisinin in the presence of $50 \mu \mathrm{M}$ vitamin $\mathrm{E}(\mathrm{g})$. The bar represents $100 \mu \mathrm{m}$. B, Quantitative evaluation of HIF- $1 \alpha$ immunofluorescence in three independent experiments under experimental conditions as indicated. In each experiment at least 20 embryoid bodies were analyzed. * $p$ $<0.05$, significantly different from the untreated control.

cent reaction product upon oxidation (Fig. 7). We observed that artemisinin (10 and $50 \mu \mathrm{M}$ ) significantly increased ROS, which was abolished in the presence of the free radical scavengers vitamin $\mathrm{E}(50 \mu \mathrm{M})$ and mannitol (10 mm) $(n=3)$.

\section{Increased Penetration of the Cell-Permeable Fluorescence Dye Doxorubicin in Artemisinin-Treated Embryoid Bodies}

Inhibition of vasculogenesis/angiogenesis in embryoid bodies by antiangiogenic agents has been previously demonstrated by us to reduce the diffusion of fluorescence dyes (Sauer et al, 2000; Wartenberg et al, 1998). To investigate the penetration of the fluorescence dye doxorubicin, control and artemisinin-treated embryoid bodies were incubated for 30 minutes with $10 \mu \mathrm{m}$ doxorubicin. The penetration of the dye was assessed in distinct depths within the tissue (Fig. 8). Although
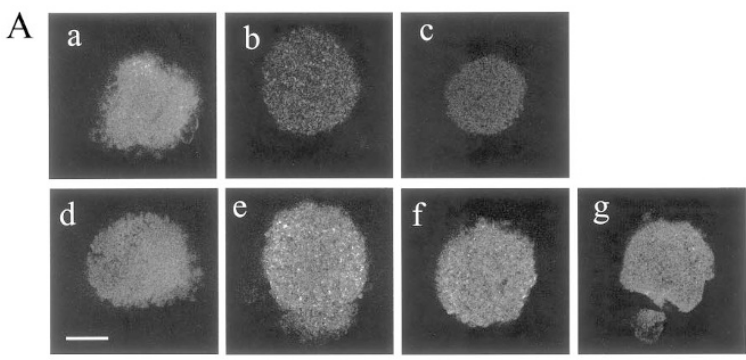

B

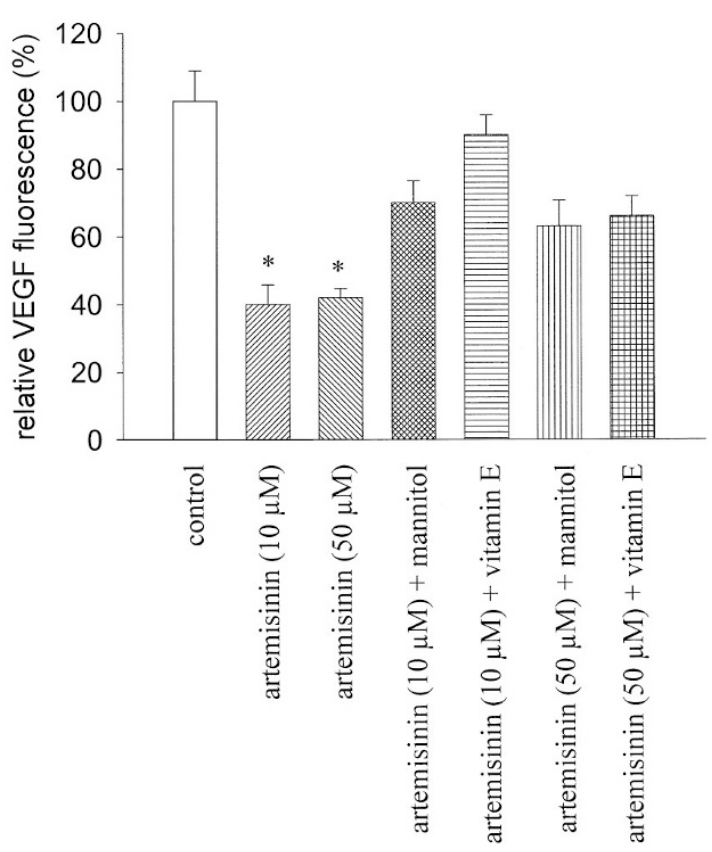

Figure 5.

Down-regulation of vascular endothelial growth factor (VEGF) expression upon treatment with artemisinin and effects of free radical scavengers. A, Representative embryoid bodies that remained untreated (control) (a) or were treated with $10 \mu \mathrm{m}$ artemisinin (b), $50 \mu \mathrm{m}$ artemisinin (c), $10 \mathrm{~mm}$ mannitol alone (d), $50 \mu \mathrm{m}$ vitamin $\mathrm{E}$ alone (e), $50 \mu \mathrm{m}$ artemisinin in the presence of 10 mm mannitol (f), or $50 \mu \mathrm{m}$ artemisinin in the presence of $50 \mu \mathrm{m}$ vitamin $\mathrm{E}(\mathrm{g})$. The bar represents $100 \mu \mathrm{m}$. B, Quantitative evaluation of VEGF immunofluorescence in three independent experiments under experimental conditions as indicated. In each experiment at least 20 embryoid bodies were analyzed. * $p$ $<0.05$, significantly different from the untreated control.

artemisinin efficiently inhibited vasculogenesis/angiogenesis in embryoid bodies (Fig. 3), improved penetration of doxorubin was observed in the treated sample, which resulted in a shift of the maximum doxorubin penetration after 30 minutes from $20 \mu \mathrm{m}$ in the untreated control to approximately $60 \mu \mathrm{m}$ in the samples treated with artemisinin $(n=3)$. Upon preincubation with $10 \mathrm{~mm}$ mannitol and $50 \mu \mathrm{M}$ vitamin $\mathrm{E}$ (data not shown), the effects of artemisinin on doxorubicin penetration were totally reversed, which further validated the assumption of a redox-mediated action of artemisinin.

\section{Disruption of the Laminin Extracellular Matrix Scaffold in Artemisinin-Treated Embryoid Bodies}

The diffusion properties of avascular tissues are limited by the formation of an extracellular matrix scaffold (Vargova et al, 2003). Hence, disruption of the extra- 


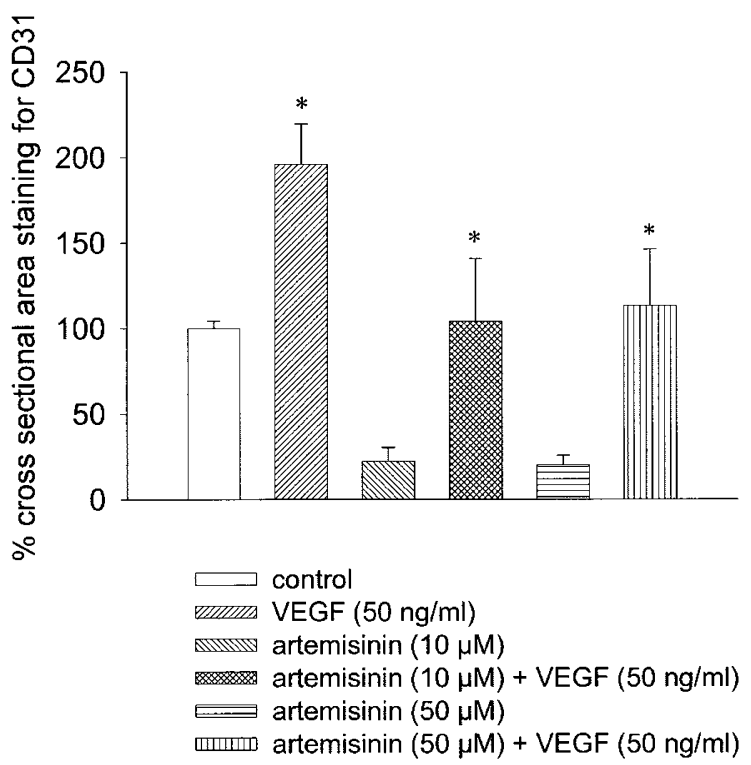

Figure 6.

Reversal of the antiangiogenic effects of artemisinin by exogenous VEGF. Embryoid bodies were treated from Day 3 through Day 8 with 10 or $50 \mu \mathrm{m}$ artemisinin either in the absence or presence of $50 \mathrm{ng} / \mathrm{ml}$ VEGF. Capillary structure formation was assessed by quantitative evaluation of the crosssectional area staining positive for CD31. Note that the antiangiogenic effect of artemisinin was reversed by coadministration of VEGF, suggesting that artemisinin impairs VEGF expression. ${ }^{*} p<0.05$, significantly different from the untreated control.

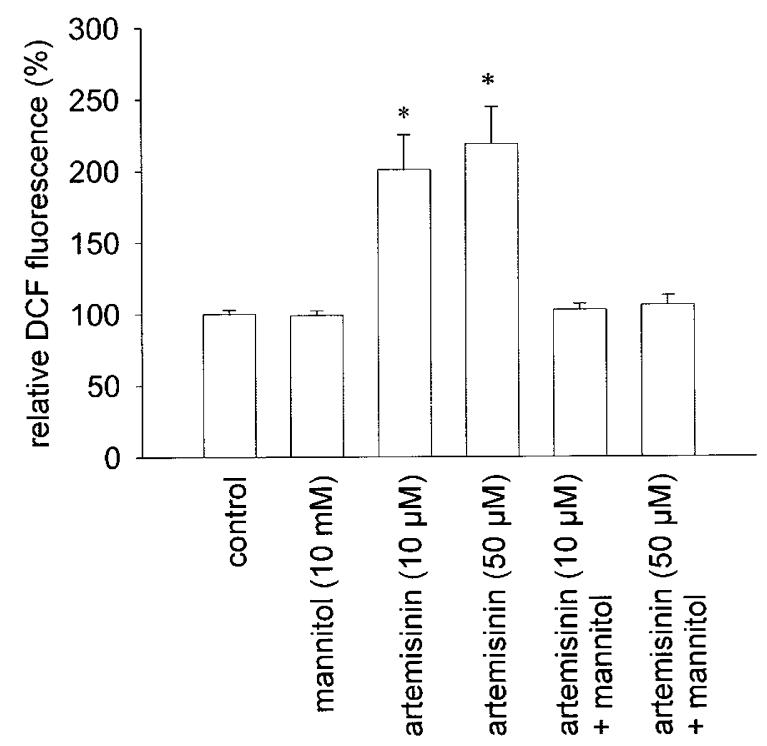

Figure 7.

Generation of reactive oxygen species (ROS) upon treatment of embryoid bodies by artemisinin. Embryoid bodies were incubated with the ROS indicator $\mathrm{H}_{2}$ DCFDA for 20 minutes. DCF fluorescence, which is indicative for intracellular ROS, was investigated by confocal laser scanning microscopy. Note that artemisinin in concentrations of 10 and $50 \mu \mathrm{m}$ significantly increased intracellular ROS, which was totally abolished in the presence of the hydroxyl radical scavenger mannitol.

cellular matrix should result in increased diffusion properties of the tissue. To investigate possible effects of artemisinin on the extracellular matrix, controls and samples treated from Day 3 to Day 8 of cell culture

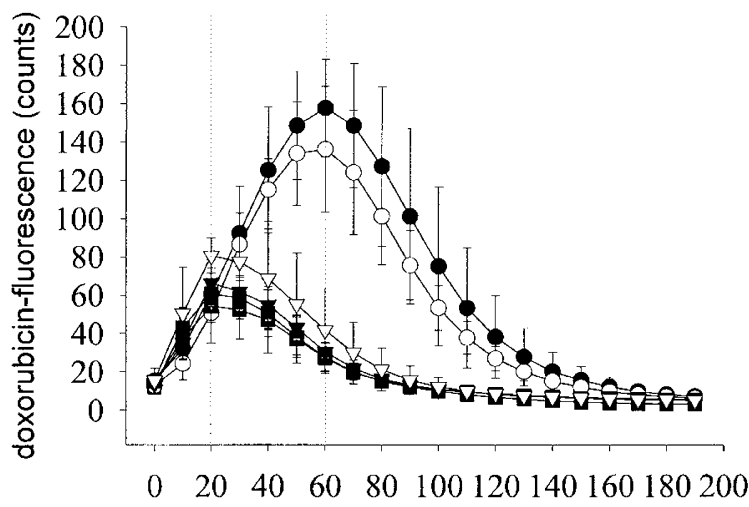

penetration depth of the laser beam $(\mu \mathrm{m})$

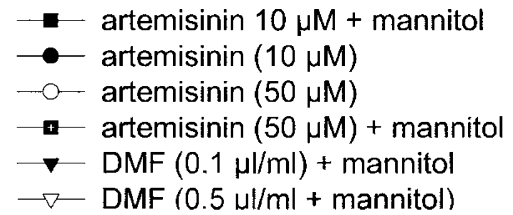

Figure 8

Increased penetration of doxorubicin upon treatment of embryoid bodies with artemisinin. Doxorubicin fluorescence was assessed by the optical probe technique (Meshnick, 1994) in distinct depths within the tissue under experimental conditions as indicated. Note that artemisinin treatment resulted in increased penetration of doxorubicin in the multicellular tissue, which was totally abolished in the presence of the free radical scavenger mannitol. The dashed vertical lines indicate the maxima of the curves.

with $50 \mu \mathrm{M}$ artemisinin were immunostained with an antibody directed against laminin, which is a major constituent of the extracellular matrix $(n=5)$ (Fig. 9). We observed that artemisinin treatment induced disruption of the laminin network, which resulted in a punctate appearance of laminin staining. In contrast, complex network-like laminin structures were obtained in the untreated control (Fig. 9, inset). Upon coadministration of either the free radical scavenger mannitol $(10 \mathrm{~mm})$ or vitamin $\mathrm{E}(50 \mu \mathrm{M})$, the artemisinininduced disruption of the laminin network was completely abolished.

\section{Altered Expression Patterns of Matrix Metalloproteinases (MMPs) in Artemisinin-Treated Embryoid Bodies}

In embryoid bodies, angiogenesis is accompanied by expression of MMPs in a strictly time-regulated manner (Wartenberg et al, 2003). Endothelial cell growth during angiogenesis requires the degradation of the extracellular matrix, the basement membrane, and the interstitial stroma and is governed by the activation of MMPs. However, it has been recently discussed that activation of MMPs, depending on their substrate (growth factors or their receptors, extracellular matrix components, and angiogenic factors), may result in the generation of proangiogenic and antiangiogenic factors, the latter resulting in an inhibition of angiogenesis (Heissig et al, 2003). The effects of artemisinin on MMP expression were assessed by histochemical analysis of MMP-1, MMP-2, and MMP-9 expression in 

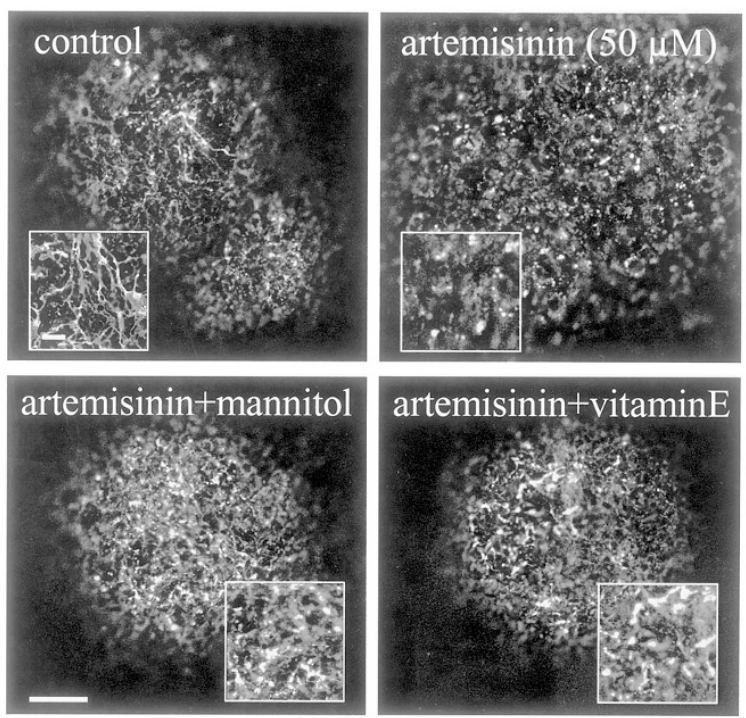

Figure 9.

Effects of artemisinin treatment on the laminin extracellular matrix scaffold. Shown are representative embryoid bodies that were treated from Day 3 through Day 8 of cell culture with $50 \mu \mathrm{m}$ artemisinin. Artemisinin treatment resulted in disruption of the laminin extracellular matrix, which had a network-like appearance in the untreated control (upper left and inset), but had a punctate appearance in the presence of artemisinin (upper right and inset). Coadministration of mannitol ( $10 \mathrm{~mm})$ (lower left) and vitamin E (50 $\mu \mathrm{M})$ (lower right) abolished the effect of artemisinin, indicating involvement of ROS. The bar in the lower left image represents $100 \mu \mathrm{m}$. The bar in the inset represents $20 \mu \mathrm{m}$.

control embryoid bodies versus embryoid bodies that were treated from Day 3 to Day 8 with $50 \mu \mathrm{m}$ artemisinin (Fig. 10). As previously shown (Wartenberg et al, 2003a) endogenous MMP-1 expression increased between Day 4 and Day 8 of cell culture $(n=3)$. Upon treatment with $50 \mu \mathrm{m}$ artemisinin, a significantly elevated expression of MMP-1, with maximum values on Day 5 of embryoid body culture, was observed (656 \pm $130 \%$ as compared with MMP-1 immunofluorescence of the untreated control on Day 5 of cell culture, which amounted to $125 \pm 16 \%)(n=3)$. Interestingly MMP-1 expression in the presence of artemisinin declined during prolonged times of cell culture, ie, Days 6 to 8 , and reached values that were significantly lower on Day 8 in the artemisinin-treated sample $(256 \pm 72)$ as compared with the untreated control $(425 \pm 19 \%)$.

The endogenous expression of MMP-2 exerted a comparable time course as compared with MMP-1 ( $n$ $=3$ ). However, in the artemisinin-treated sample, a significant increase in MMP-2 expression was observed from Day 6 to Day 8 of cell culture $(n=3)$-Day 6: $504 \pm 21 \%$, Day 7: $609 \pm 34$, and Day 8: $623 \pm 5$ in the artemisinin-treated sample versus Day 6: $281 \pm$ 23\%, Day 7: $391 \pm 11$, and Day 8: $500 \pm 18$ in the control. As previously shown (Wartenberg et al, 2003a) endogenous MMP-9 expression was lower as compared with MMP-1 and MMP-2 but increased during the time course of embryoid bodies culture to $238 \pm$ $10 \%$ (immunofluorescence at Day 4 set to 100\%) ( $n=$ 3). In contrast, treatment with $50 \mu \mathrm{M}$ artemisinin resulted in significantly elevated MMP-9 expression at Day 4 of embryoid body culture $(308 \pm 13 \%)$, which

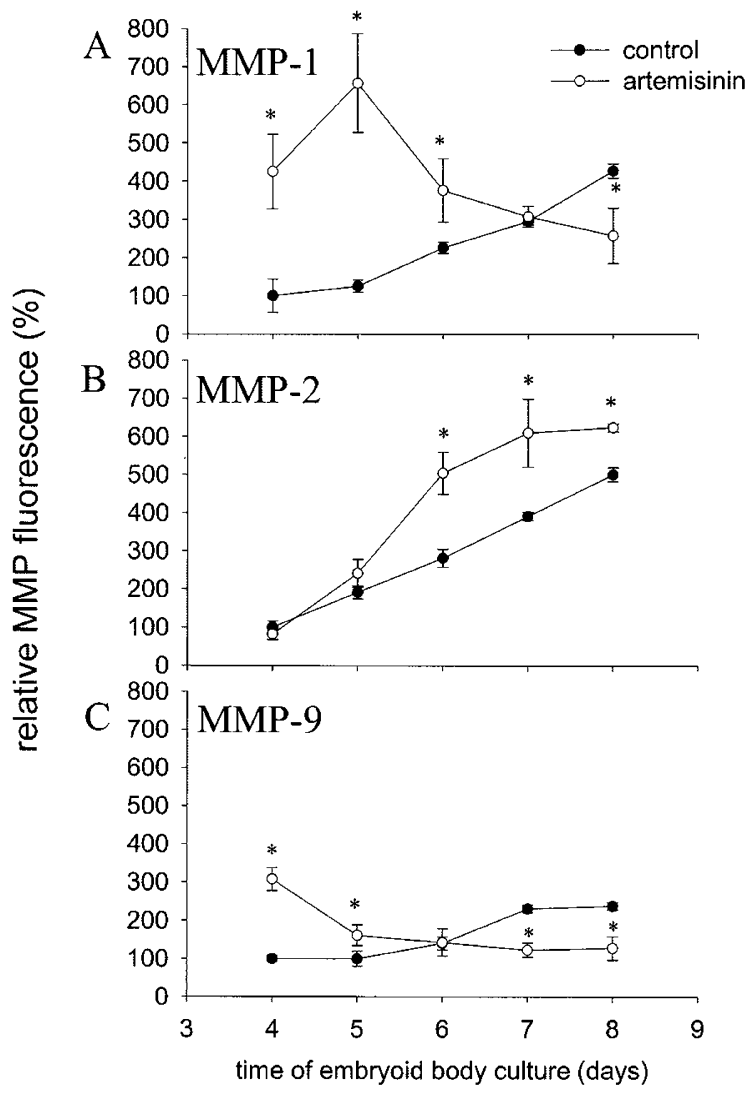

Figure 10.

Alteration of matrix metalloproteinase (MMP) expression upon treatment of embryoid bodies during differentiation with artemisinin. MMP-1 (A), MMP-2 (B), and MMP-9 (C) expression in control and artemisinin (50 $\mu \mathrm{M})$-treated whole mount embryoid bodies. Note that artemisinin treatment significantly changed the time course of MMP-1, MMP-2, and MMP-9 expression observed in the untreated control. ${ }^{*} p<0.05$, significantly different from the untreated control.

decreased to $128 \pm 15 \%$ on Day 8 of embryoid body culture $(n=3)$.

\section{Discussion}

In the present study, we report on antiangiogenic effects of the antimalaria agent artemisinin, which has recently also proven effective against several cancer cell lines (Efferth et al, 2002). Hence, our data suggest that possible future applications for artemisinin in cancer therapies may include the use of this compound as an antiangiogenic agent. Because artemisinin in the applied concentrations did not inhibit cardiomyogenesis of ES cells, an overall effect on the commitment of the mesodermal cell lineage was excluded. It should, however, be mentioned that the differentiation of other cell types could be affected by artemisinin treatment.

Our data clearly suggest that the antiangiogenic effects of artemisinin are based on free radicals generated within the tissue. Generation of ROS has been previously shown to be the basis for the antiparasitic action of artemisinin (Scott et al, 1989). In this respect it has been discussed that enhanced ROS production 
after the administration of pro-oxidants, which is directed against the intraerythrocytic parasite, inhibits the infection both in vitro and in vivo. However, it has been recently suggested that ROS are also involved in pathologic changes in host tissue-like damage of the vascular endothelial lining during a malaria infection (cerebral malaria). Pro-oxidants support the host defense against the parasite when working in or near the infected cell but potentially cause vascular damage when working on or near the vascular lining (Postma et al, 1996).

Vascular damage conferred by oxidative stress is a well-known feature, occurring during ischemia/reperfusion (Molyneux et al, 2002), arteriosclerosis (Harrison et al, 2003), and after administration of teratogenic agents such as thalidomide (Sauer et al, 2000) and valproate (Sobaniec-Lotowska and Sobaniec, 1996). Because artemisinin treatment resulted in a strong reduction of vascular growth in the embryonic tissue of ES cell-derived embryoid bodies, the application of the drug at least at high doses used in the present study should be avoided in pregnant women. Although artemisinin at low doses used for antimalaria treatment has been recently demonstrated to be well tolerated in pregnant women, and birth outcomes did not differ significantly from community rates for abortion, stillbirth, congenital abnormality, and mean gestation at delivery, further information about the safety of these valuable antimalarials in pregnancy was requested (McGready et al, 2001). This is all the more important because in mice, hamsters, guinea pigs, and rabbits, both dihydroartemisinin and artesunate showed contragestational effects (Xu et al, 1996).

The present data suggest that the antiangiogenic effects of artemisinin are at least partially based on oxidative stress-mediated reduction of HIF- $1 \alpha$ expression, with the consequence of decreased expression of VEGF, which is a key determinant of endothelial cell growth because the free radical scavengers vitamin $E$ and mannitol restored HIF- $1 \alpha$ and VEGF expression and reversed angiogenesis to the control level. These data were corroborated by experiments that showed that the antiangiogenic effects of artemisinin could be partially abolished upon addition of VEGF to the cell culture medium. The redox-regulation of $\mathrm{HIF}-1 \alpha$ is currently a matter of discussion. A number of studies have demonstrated that ubiquitination and proteasomal degradation of $\mathrm{HIF}-1 \alpha$ are stimulated in the presence of elevated ROS, eg, in the presence of extracellular added hydrogen peroxide (Huang et al, 1996; Wartenberg et al, 2003b). Other studies have demonstrated that elevation of intracellular ROS by treatment of cells with growth factors and hormones, eg, angiotensin II (Page et al, 2002), thrombin, and transforming growth factor- $\beta$ (Gorlach et al, 2001), results in increased HIF- $1 \alpha$ transcription by a process that is oxygen independent.

In addition to the observed decrease in HIF-1 $\alpha$ and VEGF expression, a dysregulation in MMP-1, -2, and -9 expression was observed in artemisinin-treated embryoid bodies during the time course of vasculogenesis/angiogenesis. Endothelial cell proliferation and migration themselves are under tight control by a balance of angiogenesis inducers and inhibitors. A large number of angiogenic factors work together in a highly coordinated manner to induce endothelial cell outgrowth and the formation of functional vessels. Therefore coordinated MMP expression may be a prerequisite for normal vascular growth, whereas deregulation of the time frame of MMP expression may result in impaired angiogenesis. The deregulation of MMP expression upon exposure of embryoid bodies to artemisinin is presumably a result of the pro-oxidant properties of this compound; it has been previously shown that exogenous addition of hydrogen peroxide (Belkhiri et al, 1997; Brenneisen et al, 1997) as well as an increase in intracellular ROS through overexpression of Mn-superoxide dismutase resulted in upregulation of several MMPs (Ranganathan et al, 2001).

The increased expression of MMP-1 and MMP-9 during early stages of embryoid body differentiation and of MMP-2 during later stages may be the cause for the disruption of the laminin-scaffold observed in artemisinin-treated samples because extracellular matrix components such as laminin, fibronectin, and collagens are the targets of MMP enzymatic activity. The extracellular matrix is the main barrier to the delivery of therapeutic agents. Therefore, degradation of matrix components should facilitate the penetration of anticancer agents, allowing an improvement in chemotherapeutic treatment. In the present study, artemisinin treatment resulted in significant increased penetration of the fluorescent anthracycline doxorubicin, which was reversed upon cotreatment with free radical scavengers. The increased penetration of doxorubicin in the three-dimensional tissue should increase the cytotoxic action of this anticancer agent in solid tumors and supplement the abrogation of tumor growth achieved by inhibition of tumor-induced angiogenesis. Because artemisinin and a number of derivatives of this compound have been recently demonstrated to exert cytostatic or cytotoxic activity against cancer cells (Efferth et al, 2001, 2002; Posner et al, 2003), these compounds, in addition to their antimalaria action, are likely fulfilling their promise as anticancer agents in the future.

\section{Materials and Methods}

\section{Spinner-Culture Technique for Cultivation of Embryoid Bodies}

The ES cell line CCE was grown on mitotically inactivated feeder layers of primary murine embryonic fibroblasts for a maximum of eight passages in Iscove's medium (Gibco, Live Technologies, Helgerman Court, Maryland) supplemented with $20 \%$ heat-inactivated (56 ${ }^{\circ} \mathrm{C}, 30$ minutes) FCS (Boehringer-Mannheim, Germany), 2 mм Glutamax (Gibco), $100 \mu \mathrm{M}$ $\beta$-mercaptoethanol (Sigma, Deisenhofen, Germany), $1 \%$ MEM nonessential amino acids stock solution (Gibco), $100 \mathrm{lU} / \mathrm{ml}$ penicillin, and $100 \mu \mathrm{g} / \mathrm{ml}$ streptomycin (both Gibco) in a humidified environment containing $5 \% \mathrm{CO}_{2}$ at $37^{\circ} \mathrm{C}$ and passaged every 2 to 3 
days. At Day 0 of differentiation, adherent cells were enzymatically dissociated using $0.2 \%$ trypsin and $0.05 \%$ EDTA in PBS (Gibco) and seeded at a density of $1 \cdot 10^{7}$ cells $\mathrm{ml}^{-1}$ in $250 \mathrm{ml}$ of siliconized spinner flasks (Integra Biosciences, Fernwald, Germany) containing $100 \mathrm{ml}$ of Iscove's medium supplemented with the same additives as described above. After 24 hours, $150 \mathrm{ml}$ of medium was added to give a final volume of $250 \mathrm{ml}$. The spinner flask medium was stirred at $20 \mathrm{rpm}$ using a stirrer system (Integra Biosciences), and $150 \mathrm{ml}$ of cell culture medium was exchanged every day.

\section{Immunohistochemistry}

Immunohistochemistry was performed with whole mount embryoid bodies. As primary antibodies, the mouse monoclonal anti-PECAM-1 (CD31) (Endogen, Woburn, Massachusetts) (concentration of $5 \mu \mathrm{g} / \mathrm{ml}$ ), the mouse monoclonal anti-Ki67 antibody (Sigma), the mouse monoclonal anti-MMP-1 (dilution 1:100) and MMP-9 (dilution 1:100) (Oncogene Research Products, Boston, Massachusetts), and the polyclonal rabbit anti-MMP-2 (dilution 1:100) (Chemicon, Temecula, California) were used. For PECAM-1 staining the respective tissues were fixed in ice-cold methanol/ acetone $(7: 3)$ for 60 minutes at $-20^{\circ} \mathrm{C}$ and washed with PBS containing $0.1 \%$ Triton $\mathrm{X}-100$ (PBST) (Sigma). For MMP and Ki-67 staining, the tissues were fixed for 1 hour at $4^{\circ} \mathrm{C}$ in $4 \%$ formaldehyde in PBS. Blocking against unspecific binding was performed for 60 minutes with $10 \%$ fat-free milk powder (Heirler, Radolfzell, Germany) dissolved in PBS. The tissues were subsequently incubated for 90 minutes (for MMP staining overnight at $8^{\circ} \mathrm{C}$ ) at room temperature with primary antibodies dissolved in PBS supplemented with $10 \%$ milk powder. The tissues were thereafter washed three times with PBST $(0.01 \%$ Triton) and reincubated with either a Cy5-conjugated rabbit antiSyrian hamster IgG $(\mathrm{H}+\mathrm{L})$ (PECAM-1), a Cy5conjugated goat anti-mouse IgG (H+L) (Ki-67, MMP-1, and MMP-9), or a Cy3-conjugated goat antirabbit IgG (MMP-2) (all from Dianova, Hamburg, Germany) at a concentration of $3.8 \mu \mathrm{g} / \mathrm{ml}$ in PBS containing $10 \%$ milk powder. After washing three times in PBST $(0.01 \%$ Triton), the tissues were stored in PBS until inspection. For the excitation of the Cy5 fluorochrome, the 633-nm band of a helium/neon laser of the confocal setup was used. Emission was recorded using a 655-nm longpass filter set. The Cy3 fluorochrome was excited by the 543-nm band of a helium/ neon laser, and emission was recorded using a 570-nm longpass filter set. The pinhole settings of the confocal setup were adjusted to give a full width half maximum of $9 \mu \mathrm{m}$. Fluorescence was recorded at a depth of 80 to $120 \mu \mathrm{m}$ in the tissue, and the fluorescence values in the respective optical section were evaluated by the image analysis software of the confocal setup.

\section{Measurement of ROS Generation}

Intracellular ROS levels were measured using the fluorescent dye $\mathrm{H}_{2}$ DCF-DA (Molecular Probes, Eugene, Oregon), which is a nonpolar compound that is converted into a nonfluorescent polar derivative $\left(\mathrm{H}_{2}\right.$ DCF) by cellular esterases after incorporation into cells. $\mathrm{H}_{2}$ DCF is membrane impermeable and is rapidly oxidized to the highly fluorescent 2',7'dichlorofluorescein (DCF) in the presence of intracellular ROS. For the experiments, embryoid bodies were incubated in $\mathrm{E} 1$ medium [containing in mM: $\mathrm{NaCl} 135$, $\mathrm{KCl} 5.4, \mathrm{CaCl}_{2}$ 1.8, $\mathrm{MgCl}_{2} 1$, glucose 10, HEPES 10 (pH 7.4 at $\left.23^{\circ} \mathrm{C}\right)$, and $20 \mu \mathrm{M} \mathrm{H} \mathrm{H}_{2}$ DCF-DA dissolved in dimethyl sulfoxide (DMSO) was added. After 20 minutes intracellular DCF fluorescence (corrected for background fluorescence) was evaluated in $3600 \mu \mathrm{m}^{2}$ regions of interest using an overlay mask unless otherwise indicated. For fluorescence excitation, the 488-nm band of the argon ion laser of the confocal setup was used. Emission was recorded using a longpass LP515-nm filter set.

\section{Assessment of Doxorubicin Penetration in Embryoid Bodies}

To assess tissue permeability after treatment with artemisinin, embryoid bodies were treated from Day 3 through Day 8 with either $10 \mu \mathrm{M}$ artemisinin, $50 \mu \mathrm{M}$ artemisinin, $50 \mu \mathrm{M}$ artemisinin in the presence of 10 $\mathrm{mm}$ mannitol, or mannitol in the presence of either 0.1 $\mu \mathrm{l} / \mathrm{ml}$ or $0.5 \mu \mathrm{l} / \mathrm{ml}$ of the solvent dimethylformamide. Subsequently they were incubated with $10 \mu \mathrm{m}$ of the fluorescent anthracycline doxorubicin (Sigma), and penetration of the dye was investigated after $30 \mathrm{~min}-$ utes in $10-\mu \mathrm{m}$ optical sections by the use of the 543-nm line of a helium/neon laser of the confocal setup and the optical probe technique as described before (Wartenberg et al, 1998b).

\section{Statistical Analysis}

Data are given as mean values \pm SEM, with $n$ denoting the number of experiments unless otherwise indicated. In each experiment at least 15 embryoid bodies were analyzed. Student's $t$ test for unpaired data was applied as appropriate. A value of $p<0.05$ was considered significant.

\section{References}

Belkhiri A, Richards C, Whaley M, McQueen SA, and Orr FW (1997). Increased expression of activated matrix metalloproteinase- 2 by human endothelial cells after sublethal $\mathrm{H}_{2} \mathrm{O}_{2}$ exposure. Lab Invest 77:533-539.

Brenneisen $\mathrm{P}$, Briviba $\mathrm{K}$, Wlaschek $\mathrm{M}$, Wenk $\mathrm{J}$, and Scharffetter-Kochanek K (1997). Hydrogen peroxide $\left(\mathrm{H}_{2} \mathrm{O}_{2}\right)$ increases the steady-state mRNA levels of collagenase/ MMP-1 in human dermal fibroblasts. Free Radic Biol Med 22:515-524.

Efferth T, Dunstan H, Sauerbrey A, Miyachi H, and Chitambar CR (2001). The anti-malarial artesunate is also active against cancer. Int J Oncol 18:767-773. 
Efferth T, Olbrich A, and Bauer R (2002). mRNA expression profiles for the response of human tumor cell lines to the antimalarial drugs artesunate, arteether, and artemether. Biochem Pharmacol 64:617-623.

Gorlach A, Diebold I, Schini-Kerth VB, BerchnerPfannschmidt $\mathrm{U}$, Roth $\mathrm{U}$, Brandes RP, Kietzmann $\mathrm{T}$, and Busse R (2001). Thrombin activates the hypoxia-inducible factor-1 signaling pathway in vascular smooth muscle cells: Role of the p22(phox)-containing NADPH oxidase. Circ Res 89:47-54.

Harrison D, Griendling KK, Landmesser U, Hornig B, and Drexler $\mathrm{H}$ (2003). Role of oxidative stress in atherosclerosis. Am J Cardiol 91:7A-11A.

Haynes RK (2001). Artemisinin and derivatives: The future for malaria treatment? Curr Opin Infect Dis 14:719-726.

Heissig B, Hattori K, Friedrich M, Rafii S, and Werb Z (2003). Angiogenesis: Vascular remodeling of the extracellular matrix involves metalloproteinases. Curr Opin Hematol 10:136-141.

Huang LE, Arany Z, Livingston DM, and Bunn HF (1996). Activation of hypoxia-inducible transcription factor depends primarily upon redox-sensitive stabilization of its alpha subunit. J Biol Chem 271:32253-32259.

Jefford CW (2001). Why artemisinin and certain synthetic peroxides are potent antimalarials: Implications for the mode of action. Curr Med Chem 8:1803-1826.

Mazure NM, Brahimi-Horn MC, and Pouyssegur J (2003). Protein kinases and the hypoxia-inducible factor-1, two switches in angiogenesis. Curr Pharm Des 9:531-541.

McGready R, Cho T, Keo NK, Thwai KL, Villegas L, Looareesuwan S, White NJ, and Nosten F (2001). Artemisinin antimalarials in pregnancy: A prospective treatment study of 539 episodes of multidrug-resistant Plasmodium falciparum. Clin Infect Dis 33:2009-2016.

Meshnick SR (1994). Free radicals and antioxidants. Lancet 344:1441-1442.

Meshnick SR, Taylor TE, and Kamchonwongpaisan S (1996). Artemisinin and the antimalarial endoperoxides: From herbal remedy to targeted chemotherapy. Microbiol Rev 60:301315.

Molema G (2002). Tumor vasculature directed drug targeting: Applying new technologies and knowledge to the development of clinically relevant therapies. Pharm Res 19:12511258.

Molyneux CA, Glyn MC, and Ward BJ (2002). Oxidative stress and cardiac microvascular structure in ischemia and reperfusion: The protective effect of antioxidant vitamins. Microvasc Res 64:265-277.

Page EL, Robitaille GA, Pouyssegur J, and Richard DE (2002). Induction of hypoxia-inducible factor-1alpha by transcriptional and translational mechanisms. J Biol Chem 277: 48403-48409.

Posner GH, Paik IH, Sur S, McRiner AJ, Borstnik K, Xie S, and Shapiro TA (2003). Orally active, antimalarial, anticancer, artemisinin-derived trioxane dimers with high stability and efficacy. J Med Chem 46:1060-1065.

Posner GH, Ploypradith P, Parker MH, O'Dowd H, Woo SH, Northrop J, Krasavin M, Dolan P, Kensler TW, Xie S, and Shapiro TA (1999). Antimalarial, antiproliferative, and antitumor activities of artemisinin-derived, chemically robust, trioxane dimers. J Med Chem 42:4275-4280.
Postma NS, Mommers EC, Eling WM, and Zuidema J (1996). Oxidative stress in malaria: Implications for prevention and therapy. Pharm World Sci 18:121-129.

Ranganathan AC, Nelson KK, Rodriguez AM, Kim KH, Tower GB, Rutter JL, Brinckerhoff CE, Huang TT, Epstein CJ, Jeffrey JJ, and Melendez JA (2001). Manganese superoxide dismutase signals matrix metalloproteinase expression via $\mathrm{H}_{2} \mathrm{O}_{2}$-dependent ERK1/2 activation. J Biol Chem 276: 14264-14270.

Robert A, Dechy-Cabaret O, Cazelles J, and Meunier B (2002). From mechanistic studies on artemisinin derivatives to new modular antimalarial drugs. Acc Chem Res 35:167174.

Sauer H, Gunther J, Hescheler J, and Wartenberg M (2000). Thalidomide inhibits angiogenesis in embryoid bodies by the generation of hydroxyl radicals. Am J Pathol 156:151-158.

Scott MD, Meshnick SR, Williams RA, Chiu DT, Pan HC, Lubin BH, and Kuypers FA (1989). Qinghaosu-mediated oxidation in normal and abnormal erythrocytes. J Lab Clin Med 114:401-406.

Seiler A, Visan A, Pohl I, Genschow E, Buesen R, and Spielmann H (2002). Improving the embryonic stem cell test (EST) by establishing molecular endpoints of tissue specific development using murine embryonic stem cells (D3 cells). ALTEX 2002, 19(Suppl 1):55-63.

Sobaniec-Lotowska ME and Sobaniec W (1996). Morphological features of encephalopathy after chronic administration of the antiepileptic drug valproate to rats: A transmission electron microscopic study of capillaries in the cerebellar cortex. Exp Toxicol Pathol 48:65-75.

Vargova L, Homola A, Zamecnik J, Tichy M, Benes V, and Sykova E (2003). Diffusion parameters of the extracellular space in human gliomas. Glia 42:77-88.

Vittet D, Prandini MH, Berthier R, Schweitzer A, MartinSisteron H, Uzan G, and Dejana E (1996). Embryonic stem cells differentiate in vitro to endothelial cells through successive maturation steps. Blood 88:3424-3431.

Wartenberg M, Budde P, De Marees M, Grunheck F, Tsang SY, Huang Y, Chen ZY, Hescheler J, and Sauer H (2003a). Inhibition of tumor-induced angiogenesis and matrixmetalloproteinase expression in confrontation cultures of embryoid bodies and tumor spheroids by plant ingredients used in traditional chinese medicine. Lab Invest 83:87-98.

Wartenberg M, Gunther J, Hescheler J, and Sauer H (1998a). The embryoid body as a novel in vitro assay system for antiangiogenic agents. Lab Invest 78:1301-1314.

Wartenberg M, Hescheler J, Acker H, Diedershagen $\mathrm{H}$, and Sauer H (1998b). Doxorubicin distribution in multicellular prostate cancer spheroids evaluated by confocal laser scanning microscopy and the "optical probe technique." Cytometry $31: 137-145$.

Wartenberg M, Ling FC, Muschen M, Klein F, Acker $H$, Gassmann M, Petrat K, Putz V, Hescheler J, and Sauer H (2003b). Regulation of the multidrug resistance transporter P-glycoprotein in multicellular tumor spheroids by hypoxiainducible factor (HIF-1) and reactive oxygen species. FASEB J 17:503-505.

Xu JH and Zhang YP (1996). (1996). Contragestational effects of dihydroartemisinin and artesunate. Yao Xue Xue Bao 31:657-661. 